\title{
Wild-type p53 controls the level of fibronectin expression in breast cancer cells
}

\author{
DAEUN YOU ${ }^{1 *}$, SEUNG PIL JUNG ${ }^{3 *}$, YISUN JEONG $^{1}$, SOO YOUN BAE ${ }^{3}$ and SANGMIN KIM $^{2}$ \\ ${ }^{1}$ Department of Health Sciences and Technology, SAIHST, Sungkyunkwan University; ${ }^{2}$ Department of Breast Cancer Center, \\ Samsung Medical Center, Gangnam-gu, Seoul 06351; ${ }^{3}$ Division of Breast and Endocrine Surgery, Department of Surgery, \\ Korea University Hospital, Korea University College of Medicine, Seongbuk-gu, Seoul 02852, Republic of Korea
}

Received March 16, 2017; Accepted May 29, 2017

DOI: $10.3892 /$ or.2017.5860

\begin{abstract}
Aberrant fibronectin (FN) expression is associated with poor prognosis, cell adhesion, and cell motility in a variety of cancer cells. In this study, we investigated the relationship between p53 and FN expression in breast cancer cells. Basal FN expression was significantly decreased by treatment with the p53 activator III, RITA, in MCF7 breast cancer cells with wild-type p53. In addition, overexpression of wild-type p53 markedly decreased the level of FN expression in p53-mutant breast cancer cells. To examine the mechanism underlying the relationship between p53 and FN expression, we treated MCF7 breast cancer cells with the tumor promoter TPA (12-O-tetradecanoylphorbol-13-acetate). Our results showed that basal FN expression was increased by TPA treatment in a time-dependent manner. In contrast, the level of p53 expression was decreased by TPA treatment. However, the expression of FN and p53 was not altered by TPA in p53-mutant breast cancer cells. Furthermore, the alterations in FN and p53 expression in response to TPA were prevented by a specific MEK inhibitor, UO126. Finally, we demonstrated that TPA triggers degradation of p53 through the proteasomal pathway in MCF7 cells. TPA-induced FN expression was decreased by the proteasome inhibitor MG132. Under the same condition, p53 protein expression, but not mRNA expression, was reversed by MG132. Taken together, our data demonstrate that the level of FN expression is associated with the status and expression of p53 in breast cancer cells.
\end{abstract}

\section{Introduction}

Fibronectin (FN) is one of the most abundant adhesive glycoproteins of the extracellular matrix and acts as a substrate for

Correspondence to: Dr Sangmin Kim, Department of Breast Cancer Center, Samsung Medical Center, 50 Irwon-dong, Gangnam-gu, Seoul 06351, Republic of Korea

E-mail: sangmin3005.kim@samsung.com

*Contributed equally

Key words: fibronectin, p53, MEK, ERK, proteasomal degradation cell adhesion during wound healing and metastasis (1). The complex of integrin and FN can have modulating effects on various signaling pathways including focal adhesion kinase (FAK), signal transducer and activator of transcription-3 (STAT-3), and mitogen-activated protein kinase (MAPK) pathways that promote cell invasion and metastasis in multiple cancer cells (2-4). The induction of FN by HER2 overexpression triggers cell adhesion and invasion capacities in breast cancer cells (5). In addition, excessive FN expression is linked to poor metastatic-free survival and overall survival in breast cancer patients $(6,7)$.

The tumor suppressor protein p53 is a transcription factor that can trigger cell cycle arrest, replicative senescence, or apoptosis (8). The induction of p53 expression by a wide variety of cellular stresses such as hypoxia, UV, and cytotoxic drugs is involved in accelerated cellular senescence as well as G1/S and G2/M cell cycle checkpoint activation (9-11). The TP53 gene encoding p53 is the most frequently inactivated gene in human malignancy, and somatic missense mutations of p53 are found in $~ 50 \%$ of human cancers (12). Mutations in the TP53 gene result in loss of wild-type p53 tumor suppressor functions (13). Wild-type p53 upregulates p21, the negative regulator of cyclin-dependent kinases (cdks), whereas mutant p53 enhances cdk1 and increases cell proliferation and survival (13).

The effect of p53 status on FN in breast cancer cells is not fully understood. In this study, we investigated the role of p53 on FN expression in p53 wild-type and mutant human breast cancer cells. Specifically, we examined how p53 expression is regulated by the tumor promoter TPA and whether the alteration of p53 expression by TPA affects FN expression. We found that the level of wild-type p53 expression regulated the basal and TPA-induced FN expression levels in breast cancer cells.

\section{Materials and methods}

Reagents. Dulbecco's modified Eagle's medium (DMEM) was purchased from Thermo Scientific (Hemel Hempstead, UK). Fetal bovine serum (FBS) was purchased from Hyclone (Logan, UT, USA). Phenol red-free DMEM, penicillin $(100 \mathrm{U} / \mathrm{ml})$, and $100 \mathrm{mg} / \mathrm{ml}$ streptomycin were purchased from Life Technologies (Rockville, MD, USA). MG132 was 
purchased from Sigma-Aldrich (St. Louis, MO, USA), UO126, SP600125, and Stattic were purchased from Tocris (Ellisville, MO, USA). RITA (p53 activator III), secondary horseradish peroxidase (HRP)-conjugated antibodies, and mouse monoclonal anti- $\beta$-actin antibody were purchased from Santa Cruz Biotechnology, Inc. (Santa Cruz, CA, USA). Anti-fibronectin was purchased from Abcam (Cambridge, UK). Antibodies against total (t) and phospho (p) ERK, JNK, and STAT3 were purchased from Cell Signaling Technology (Beverly, MA, USA). ECL Western Blotting Detection reagents (West-Q Chemiluminescent Substrate Plus kit) were obtained from Genedepot (Barker, TX, USA).

Cell culture. MCF7 (p53 wild-type), Hs578T (p53 mutant V157F), and BT549 (p53 mutant R249S) human breast cancer cells were grown in a humidified atmosphere of $95 \%$ air and $5 \% \mathrm{CO}_{2}$ at $37^{\circ} \mathrm{C}$ in DMEM supplemented with $10 \% \mathrm{FBS}, 2 \mathrm{mM}$ glutamine, $100 \mathrm{IU} / \mathrm{ml}$ penicillin, and $100 \mu \mathrm{g} / \mathrm{ml}$ streptomycin. Each cell line was maintained in culture medium without FBS for $24 \mathrm{~h}$ before experiments.

Western blotting. To determine the expression of secreted FN in culture media, we analyzed equal aliquots of conditioned culture media from equal numbers of cells. Cell lysates were prepared to detect total (t) and phosphor (p)-ERK, -JNK, and -STAT3, FN, p53, and $\beta$-actin expression. Equal amounts of protein $(50 \mu \mathrm{g})$ were boiled for $5 \mathrm{~min}$ in Laemmli sample buffer and then electrophoresed in $10 \%$ sodium dodecyl sulfate polyacrylamide (SDS-PAGE) gels. Separated proteins were transferred to polyvinylidene fluoride (PVDF) membranes, and the membranes were blocked with $10 \%$ skim milk in Tris-buffered saline (TBS) containing 0.01\% Tween-20 (TBS/T) for $15 \mathrm{~min}$. Blots were washed three times in TBS/T and then incubated with antibodies against t- or p-ERK, p-JNK, and p-STAT3, FN, p53, and $\beta$-actin in TBS/T buffer at $4^{\circ} \mathrm{C}$ overnight. Blots were washed three times in TBS/T and subsequently incubated with secondary HRP-conjugated antibodies (1:2,000 dilutions) in TBS/T buffer. After 1-h incubation at room temperature (RT), blots were washed three times in TBS/T, and positive immunoreactive proteins were detected using the West-Q Chemiluminescent Substrate Plus kit.

Real-time PCR. Total RNA was extracted from cells using TRIzol reagent (Invitrogen, Carlsbad, CA, USA) according to the manufacturer's protocol. Isolated RNA samples were then used for RT-PCR. Samples of total RNA $(1 \mu \mathrm{g})$ were reverse transcribed into cDNA in $20-\mu 1$ reaction volumes using a first-strand cDNA synthesis kit for RT-PCR, according to the manufacturer's instructions (MBI Fermentas, Hanover, MD, USA). Gene expression levels were quantified by real-time PCR using a SensiMix SYBR kit (Bioline Ltd., London, UK) and $100 \mathrm{ng}$ of cDNA per reaction. The primer sequences used for this analysis were as follows: human p53 (forward, 5'-GGC CCA CTT CAC CGT ACT AA-3'; reverse, 5'-AAG CGA GAC CCA GTC TCA AA-3'); human FN (forward, 5'-CCA CCC CCA TAA GGC ATA GG-3'; reverse, 5'-GTA GGG GTC AAA GCA CGA GTC ATC-3'), and GAPDH as an internal control (forward, 5'-ATT GTT GCC ATC AAT GAC CC-3'; reverse, 5'-AGT AGA GGC AGG GAT GAT GT-3'). An
Table I. p53 status in breast cancer cells.

\begin{tabular}{llcl}
\hline $\begin{array}{l}\text { Codon } \\
\text { position }\end{array}$ & $\begin{array}{c}\text { Normal } \\
\text { (amino acid) }\end{array}$ & $\begin{array}{c}\text { Mutant } \\
\text { (amino acid) }\end{array}$ & Cell name \\
\hline- & - & - & MCF7 \\
157 & GTC (Val) & TTC (Phe) & Hs578T \\
249 & AGG (Arg) & AGC (Ser) & BT549 \\
\hline
\end{tabular}

annealing temperature of $60^{\circ} \mathrm{C}$ was used for all primers. PCR was performed in a standard 384-well plate format with an ABI 7900HT real-time PCR detection system (Foster City, CA, USA). For data analysis, the raw threshold cycle $\left(\mathrm{C}_{T}\right)$ value was first normalized to the housekeeping gene for each sample to obtain a $\Delta \mathrm{C}_{T}$. The normalized $\Delta \mathrm{C}_{T}$ was then calibrated to control cell samples and obtain $\Delta \Delta \mathrm{C}_{T}$ values.

Adenovirus induction. Adenovirus expressing Lac $\mathrm{Z}$ and human p53 cDNA (Ad-p53) was a gift from Dr Hyunil Ha (Korea Institute of Oriental Medicine, Daejeon, Korea). Recombinant adenovirus expressing human p53 was reproduced in 293A cells. Expression of this construct was confirmed by western blotting. Each construct was transfected into Hs578T and BT549 cells for $24 \mathrm{~h}$ and incubated for $24 \mathrm{~h}$ in fresh culture media. Ad-p53-overexpressing Hs578T and BT549 cells were further incubated for $24 \mathrm{~h}$ in serum-free culture media, and then cell lysates and culture medium were harvested for analysis of FN and p53 expression.

Statistical analysis. Statistical significance was determined using Student's t-test. Results are presented as means \pm SEM. All quoted P-values are two-tailed, and differences were considered statistically significant when the P-value was $<0.05$. Statistical analyses were performed using Microsoft Excel.

\section{Results}

Fibronectin expression is regulated by $p 53$ in breast cancer cells. As shown in Table I, the p53 status of breast cancer cell lines was determined from the database $h t t p: / / p 53 . f r e e$. fr/Database/Cancer_cell_lines/Breast_cancer.html, and p53 wild-type breast cancer cells (MCF7) and p53-mutant breast cancer cells (Hs578T and BT549) were selected to verify the relationship between p53 and FN expression. First, we treated MCF7 breast cancer cells with wild-type p53 and the p53 activator III RITA to disrupt the MDM2-p53 complex. After $24 \mathrm{~h}$, we harvested cell culture media and whole cell lysates for analysis of FN and p53 expression. As shown in Fig. 1A, the level of FN protein expression was decreased by RITA treatment in both culture media and cell lysates. However, the basal level of p53 protein expression was slightly increased (Fig. 1A). Under the same conditions, we analyzed the levels of FN and p53 mRNA expression. As expected, the level of FN mRNA expression was decreased $0.62 \pm 0.02$-fold by RITA relative to the control (Fig. 1B).

Next, we transfected p53-mutant breast cancer cells BT549 and Hs578T with adenovirus expressing wild-type p53. As 
A

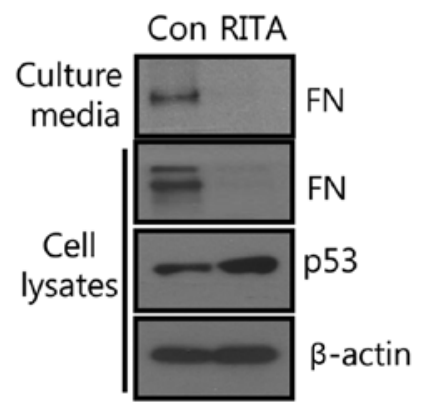

B

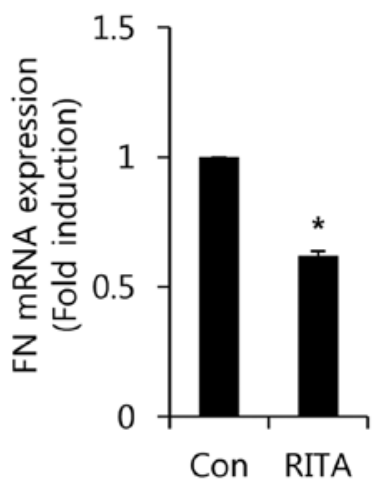

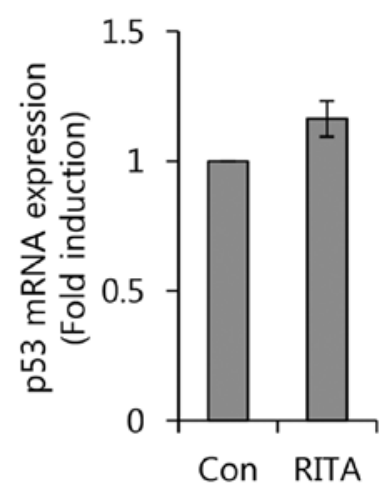

\section{C}

p53 mutant cells

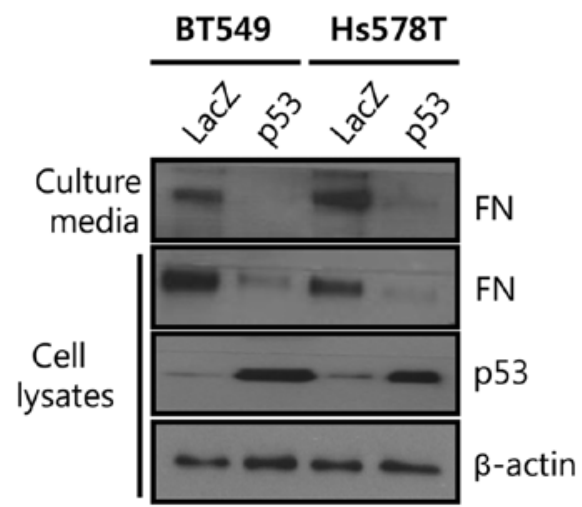

Figure 1. Fibronectin expression is regulated by p53 in breast cancer cells. (A and B) After serum starvation for $24 \mathrm{~h}$, the cells were treated with $0.5 \mu \mathrm{M}$ RITA for $9 \mathrm{~h}$. (C) p53-mutant breast cells were transfected with Ad-p53 gene for $24 \mathrm{~h}$ and further incubated for $24 \mathrm{~h}$ in fresh serum-free media. The levels of FN and p53 protein expression in culture media and cell lysates were analyzed by western blotting (A and C). The levels of FN and p53 mRNA expression were analyzed by real-time PCR (B). The results are representative of three independent experiments. Values shown are mean \pm SEM. ${ }^{*} \mathrm{P}<0.05$ vs. control. Con, control.

shown in Fig. 1C, the level of FN protein expression was significantly decreased by p53 overexpression in both BT549 and Hs578T cells. These results demonstrated that wild-type p53 expression directly influenced FN expression in breast cancer cells.

TPA reciprocally regulated the levels of $F N$ and $p 53$ expression in breast cancer cells. To verify the relationship between $\mathrm{p} 53$ and FN expression, we treated breast cancer cells with $10 \mathrm{nM}$ TPA for the indicated time. As shown in Fig. 2A, basal FN expression in MCF7 cells was increased by TPA treatment in a time-dependent manner. In contrast, the level of p53 expression was decreased from $3 \mathrm{~h}$ after TPA treatment (Fig. 2A). TPA also upregulated FN expression in ZR75-1 cells with wild-type p53 (data not shown). In addition, the level of FN mRNA expression was increased by TPA, whereas the level of p53 mRNA expression was decreased (Fig. 2B). The level of FN mRNA expression was significantly increased 6.84 \pm 1.89 -fold by treatment with $10 \mathrm{nM}$ TPA compared with the control level (Fig. 2B). As expected, the level of the p53 mRNA expression decreased $0.09 \pm 0.02$-fold compared with the control after treatment with $10 \mathrm{nM}$ TPA (Fig. 2B). However, TPA did not affect the level of FN or p53 expression in p53-mutant breast cancer cells (Fig. 2C). These results demonstrated that TPA reciprocally regulated the levels of $\mathrm{FN}$ and $\mathrm{p} 53$ expression in breast cancer cells.

Upregulation of FN and downregulation of p53 by TPA are mediated through an MEK/ERK-dependent pathway. To verify the regulatory mechanism of FN and p53 expression by TPA treatment, we examined the effect of TPA on the phosphorylation of signaling molecules ERK, JNK, and STAT3. As shown in Fig. 3, the phosphorylation of ERK and JNK was significantly increased by TPA treatment, whereas STAT3 phosphorylation was not affected.

Next, we investigated whether the MEK/ERK pathway is directly associated with the TPA-induced FN upregulation and p53 downregulation. After pretreatment with specific inhibitors for $30 \mathrm{~min}$, the cells were treated with $10 \mathrm{nM}$ TPA for 24 h. As shown in Fig. 4A, TPA-induced FN upregulation and p53 downregulation were reversed by the MEK1/2-specific inhibitor UO126 at the level of protein expression. Under the same conditions, the TPA-induced FN mRNA expression was also decreased by UO126. The level of FN mRNA increased 3.95 \pm 0.34 -fold relative to the control level after treatment with $10 \mathrm{nM}$ TPA treatment, and this effect was suppressed to $1.39 \pm 0.06$-fold by treatment with $5 \mu \mathrm{M}$ UO126 (Fig. 4B). In contrast, p53 mRNA expression was decreased 
A

p53 wild-type cells

MCF7

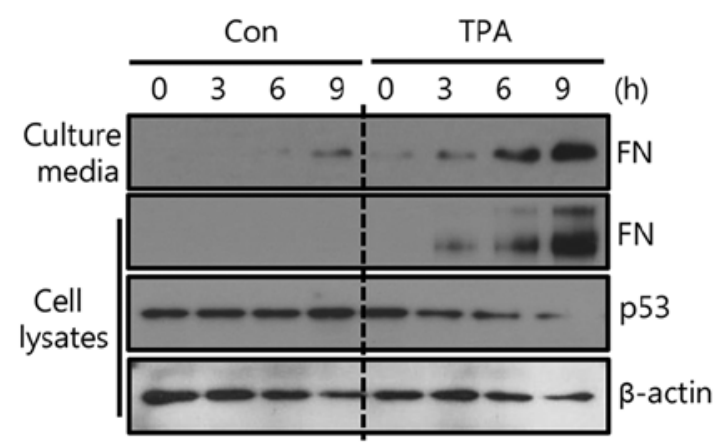

B

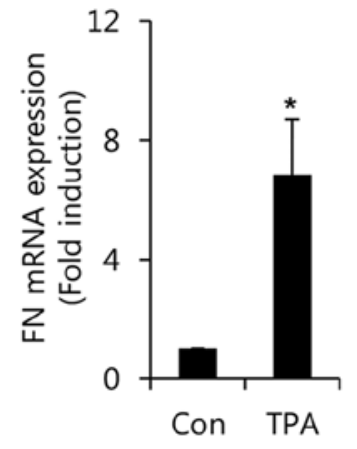

p53 wild-type cells

MCF7

\section{C p53 mutant cells}
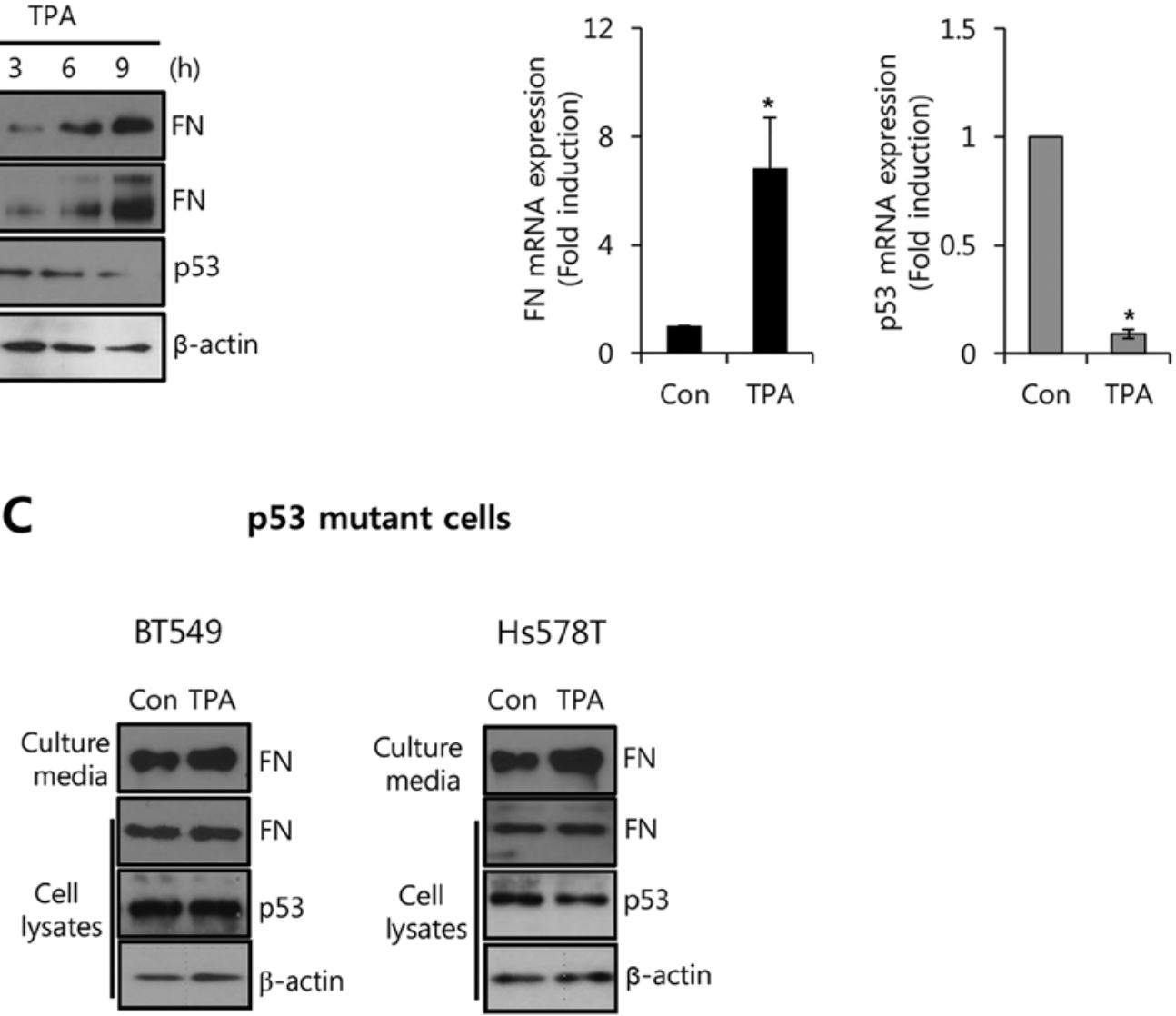

Figure 2. TPA reciprocally regulates the levels of FN and p53 expression in breast cancer cells. (A) After serum starvation for $24 \mathrm{~h}$, the cells were treated with $10 \mathrm{nM}$ TPA for the indicated time. (B and C) After serum starvation for $24 \mathrm{~h}$, the cells were treated with $10 \mathrm{nM}$ TPA for $24 \mathrm{~h}$. (A and C) Levels of FN, p53, and $\beta$-actin protein expression were analyzed by western blotting. (B) Levels of FN and p53 mRNA expression were analyzed by real-time PCR. The results are representative of three independent experiments. The values shown are the mean \pm SEM. "P $<0.05$ vs. control. Con, control.

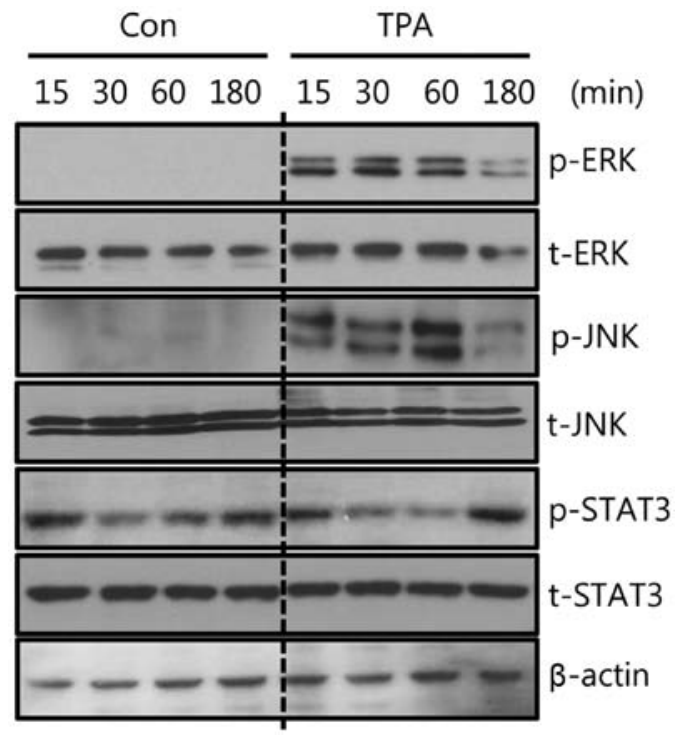

Figure 3. TPA triggers the phosphorylation of ERK and JNK, but not STAT3, in MCF7 cells. After serum starvation for $24 \mathrm{~h}, \mathrm{MCF} 7$ cells were treated with $10 \mathrm{nM}$ TPA for the indicated time. The levels of $\beta$-actin, $\mathrm{p}$ - and $\mathrm{t}$-ERK, JNK, and STAT3 protein expression were analyzed by western blotting. The results are representative of three independent experiments. Con, control. to $0.89 \pm 0.01$-fold relative to the control level by TPA, and this TPA-induced downregulation of p53 mRNA was reversed to $1.34 \pm 0.22$-fold relative to the control by treatment with $5 \mu \mathrm{M}$ UO126 (Fig. 4B). However, JNK or STAT3 signaling pathway did not affect TPA-induced FN expression (Fig. 4). These results demonstrated that the upregulation of FN and downregulation of p53 by TPA are mediated by the MEK/ ERK-dependent pathway.

Degradation of p53 by TPA upregulates the level of FN expression in MCF7 cells with wild-type p53. In a previous study, TPA stimulated the ubiquitination and degradation of p53 through downregulation of PKC- $\delta$ (14). We therefore investigated whether TPA-induced FN expression is regulated by the degradation of p53 in MCF7 cells. As shown in Fig. 5A, TPA-induced FN protein expression was significantly decreased by MG132, a proteasomal inhibitor. Conversely, the reduction of p53 protein by TPA was reversed by MG132 (Fig. 5A). Furthermore, we investigated the levels of FN and p53 mRNA expression after treatment with TPA and/or MG132. As expected, TPA-induced FN mRNA expression was decreased to $1.40 \pm 0.67$-fold relative to the control (Fig. 5B). However, the TPA-induced reduction of p53 mRNA 
A

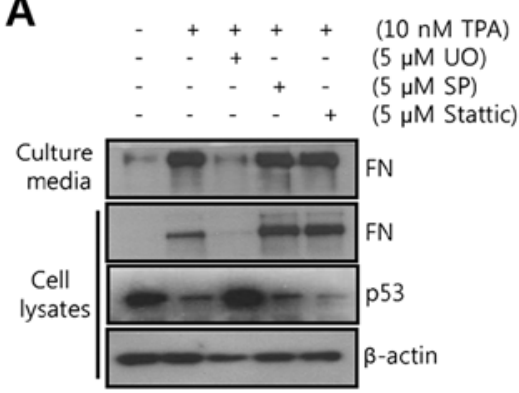

B

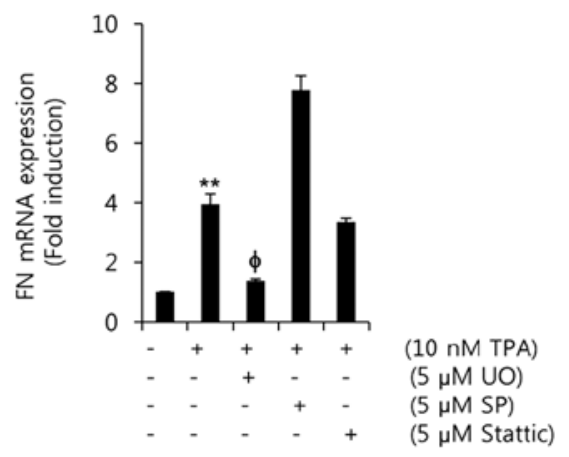

C

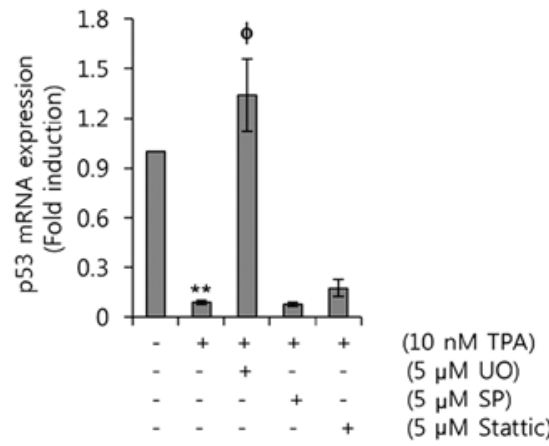

Figure 4. Upregulation of FN and downregulation of p53 by TPA are mediated by the MEK/ERK-dependent pathway. After serum starvation for 24 h, MCF7 cells were pretreated with $5 \mu \mathrm{M}$ UO, SP, and Stattic, respectively, for $30 \mathrm{~min}$ and then treated with $10 \mathrm{nM}$ TPA for $24 \mathrm{~h}$. (A) Levels of FN, p53, and $\beta$-actin protein expression were analyzed by western blotting. (B and C) Levels of FN and p53 mRNA expression were analyzed by real-time PCR. The results are representative of three independent experiments. Values shown are mean \pm SEM. ${ }^{* *} \mathrm{P}<0.01$ vs. control; ${ }^{\phi} \mathrm{P}<0.05$ vs. TPA-treated cells. Con, control; UO, UO126; SP, SP600125.

A

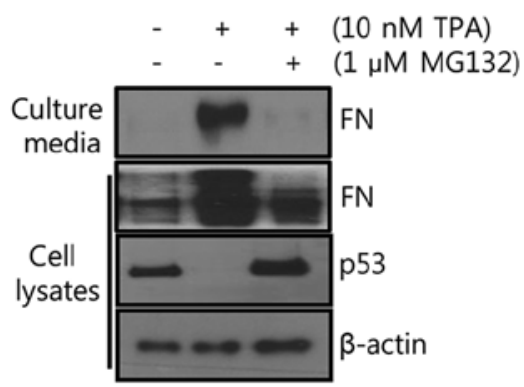

B

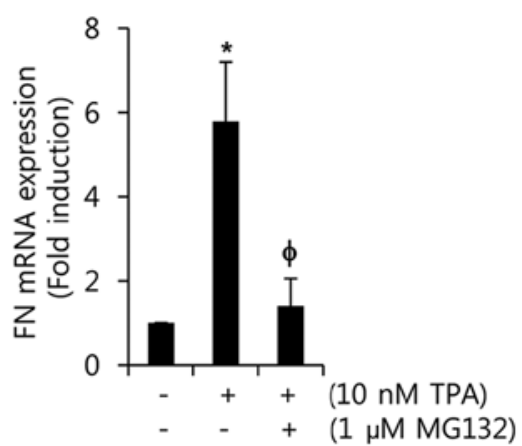

C

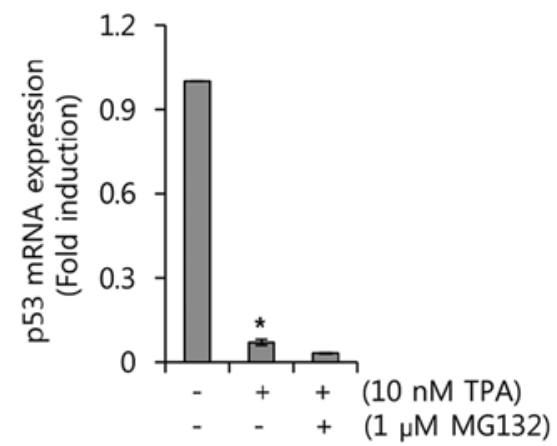

Figure 5. Degradation of p53 induced by TPA upregulates the level of FN expression in MCF7 cells with wild-type p53. After serum starvation for $24 \mathrm{~h}$, MCF7 cells were pretreated with $1 \mu \mathrm{M}$ MG132 for $30 \mathrm{~min}$ and then treated with $10 \mathrm{nM}$ TPA for $24 \mathrm{~h}$. (A) Levels of FN, p53, and $\beta$-actin protein expression were analyzed by western blotting. (B and C) Levels of FN and p53 mRNA expression were analyzed by real-time PCR. The results are representative of three independent experiments. Values shown are mean \pm SEM. ${ }^{*} \mathrm{P}<0.05$ vs. control; ${ }^{\phi}<0.05$ vs. TPA-treated cells. Con, control.

expression was not recovered by MG132 (Fig. 5C). Based on our results, we conclude that TPA triggers the degradation and transcriptional suppression of p53. In addition, the alteration of p53 level by TPA is associated with the level of FN expression in p53 wild-type breast cancer cells.

\section{Discussion}

FN is markedly upregulated in the malignant breast cancer cells but not in non-malignant breast epithelial cells $(15,16)$. Abnormal FN expression in carcinoma cells is associated with tumor aggressiveness and poor prognoses in patients with invasive breast cancer (6,7). The binding of FN/ $\alpha 5 \beta 1$ integrin augments the invasiveness of breast and ovarian cancer cells through c-Met/FAK/Src signaling pathway $(15,17)$. In addition, FN contributes to endocrine resistance through interaction with $\beta 1$ integrin and then stimulates PI3K/Akt and MAPK/ERK1/2 signaling pathway in ER- $\alpha$ positive breast cancer cells (18). Recently, we also reported that the FN-induced adhesion and invasion characteristics of breast cancer cells are suppressed by an FN inhibitor (RGD tetrapeptide) (5). Here, we explored whether p53 regulates FN expression in breast cancer cells.

p53 is a tumor suppressor gene that is stimulated by cellular stress including hypoxia, ultraviolet radiation, and oxidative stress (9-11). In addition, p53 is a known transcription factor that induces the expression of various genes associated with apoptosis, cell cycle arrest, and DNA repair (19). RITA (reactivation of p53 and induction of tumor cell apoptosis) is a small molecule that binds to the $\mathrm{N}$-terminus of the $\mathrm{p} 53$ protein and thereby induces conformational changes within the molecule that prevent its association with MDM2 $(20,21)$. RITA can activate p53 downstream targets in both p53 wild-type and p53-mutant cells in a variety of cancers such as breast, fibrosarcoma, colon, and lung carcinoma (22-24). In accordance with these reports, we found that activation of p53 by RITA stimulated the downregulation of FN expression in breast cancer cells with wild-type p53. In addition, the basal level of FN expression was decreased by overexpression of wildtype p53 in p53-mutant breast cancer cells. Therefore, we demonstrated that the level of wild-type p53 expression plays an important role in FN expression in breast cancer cells. 
Phorbol esters such as TPA are well-known tumor promoters and augment tumor invasion and migration through the induction of MMP-1 and MMP-9 (25-27). TPA treatment is directly correlated with increase in p53 and PTEN nuclear translocation (28). We found that TPA time-dependently decreased the level of p53 expression in breast cancer cells with wild-type p53, but not in p53-mutant breast cancer cells. Previously, Abbas et al reported that the transcriptional regulation of $\mathrm{p} 53$ by TPA treatment is mediated by protein kinase $\mathrm{C} \delta$ (14). In this study, we found that UO126 (a specific MEK inhibitor) controls the downregulation of p53 and upregulation of FN by TPA treatment in wild-type p53 cells. In addition, we also observed that JNK or STAT3 signaling pathway could not regulate TPA-induced FN expression. Therefore, our data demonstrate that the transcriptional level of p53 in response to TPA is mediated by an MEK/ERK-dependent pathway in p53 wild-type breast cancer cells.

In response to DNA damage, $\mathrm{p} 53$ protein is post-translationally modified, triggering its degradation through the ubiquitin proteasome pathway (29). Rottlerin, a PKC- $\delta$ inhibitor, is able to prevent p53 accumulation induced by proteasome inhibition in ML-1 human thyroid cancer cells (14). Therefore, we investigated the effect of the proteasome inhibitor MG132 on the TPA-induced reduction of p53 and induction of FN expression in p53 wild-type breast cancer cells. As expected, our results showed that TPA-induced p53 degradation was prevented by proteasome inhibition. In contrast, TPA-induced FN expression was significantly decreased by MG132 treatment. These data demonstrate that the level of wild-type p53 expression is directly involved in FN expression in breast cancer cells.

In conclusion, wild-type p53 expression directly regulates the level of FN expression in breast cancer cells. Our results showed that RITA suppresses the levels of FN mRNA and protein expression. In addition, overexpression of wild-type p53 decreases FN expression in p53-mutant breast cancer cells. We also showed that TPA triggers MEK/ERK-dependent p53 transcriptional suppression and proteasomal-dependent degradation. Furthermore, the TPA-induced downregulation of p53 is associated with the upregulation of FN in p53 wild-type breast cancer cells. Therefore, we demonstrate that wild-type p53 expression is directly associated with the level of FN expression in breast cancer cells.

\section{Acknowledgements}

This study was supported by the Basic Science Research Program through the National Research Foundation of Korea (NRF) funded by the Ministry of Education (2016R1D1A1B01010508) and by the National Research Foundation of Korea (NRF) Grant funded by the Korean Government (MSIP) (2016R1A5A2945889).

\section{References}

1. Kornblihtt AR and Gutman A: Molecular biology of the extracellular matrix proteins. Biol Rev Camb Philos Soc 63: 465-507, 1988.

2. Balanis N, Wendt MK, Schiemann BJ, Wang Z, Schiemann WP and Carlin CR: Epithelial to mesenchymal transition promotes breast cancer progression via a fibronectin-dependent STAT3 signaling pathway. J Biol Chem 288: 17954-17967, 2013.

3. Guan JL: Role of focal adhesion kinase in integrin signaling. Int J Biochem Cell Biol 29: 1085-1096, 1997.
4. Qian P, Zuo Z, Wu Z, Meng X, Li G, Wu Z, Zhang W, Tan S, Pandey V, Yao Y, et al: Pivotal role of reduced let-7g expression in breast cancer invasion and metastasis. Cancer Res 71: 6463-6474, 2011

5. Jeon M, Lee J, Nam SJ, Shin I, Lee JE and Kim S: Induction of fibronectin by HER2 overexpression triggers adhesion and invasion of breast cancer cells. Exp Cell Res 333: 116-126, 2015.

6. Fernandez-Garcia B, Eiró N, Marín L, González-Reyes S, González LO, Lamelas ML and Vizoso FJ: Expression and prognostic significance of fibronectin and matrix metalloproteases in breast cancer metastasis. Histopathology 64: 512-522, 2014.

7. Bae YK, Kim A, Kim MK, Choi JE, Kang SH and Lee SJ: Fibronectin expression in carcinoma cells correlates with tumor aggressiveness and poor clinical outcome in patients with invasive breast cancer. Hum Pathol 44: 2028-2037, 2013.

8. Woods DB and Vousden KH: Regulation of p53 function. Exp Cell Res 264: 56-66, 2001.

9. Sugrue MM, Shin DY, Lee SW and Aaronson SA: Wild-type p53 triggers a rapid senescence program in human tumor cells lacking functional p53. Proc Natl Acad Sci USA 94: 9648-9653, 1997.

10. Royds JA and Iacopetta B: p53 and disease: When the guardian angel fails. Cell Death Differ 13: 1017-1026, 2006.

11. Sur S, Pagliarini R, Bunz F, Rago C, Diaz LA Jr, Kinzler KW, Vogelstein B and Papadopoulos N: A panel of isogenic human cancer cells suggests a therapeutic approach for cancers with inactivated p53. Proc Natl Acad Sci USA 106: 3964-3969, 2009.

12. Petitjean A, Mathe E, Kato S, Ishioka C, Tavtigian SV, Hainaut $P$ and Olivier M: Impact of mutant p53 functional properties on TP53 mutation patterns and tumor phenotype: Lessons from recent developments in the IARC TP53 database. Hum Mutat 28: 622-629, 2007.

13. Di Agostino S, Strano S, Emiliozzi V, Zerbini V, Mottolese M, Sacchi A, Blandino G and Piaggio G: Gain of function of mutant p53: The mutant p53/NF-Y protein complex reveals an aberrant transcriptional mechanism of cell cycle regulation. Cancer Cell 10: 191-202, 2006

14. Abbas T, White D, Hui L, Yoshida K, Foster DA and Bargonetti J: Inhibition of human p53 basal transcription by down-regulation of protein kinase Cdelta. J Biol Chem 279: 9970-9977, 2004.

15. Nam JM, Onodera Y, Bissell MJ and Park CC: Breast cancer cells in three-dimensional culture display an enhanced radioresponse after coordinate targeting of integrin alpha5betal and fibronectin. Cancer Res 70: 5238-5248, 2010.

16. Huang L, Cheng HC, Isom R, Chen CS, Levine RA and Pauli BU: Protein kinase Cepsilon mediates polymeric fibronectin assembly on the surface of blood-borne rat breast cancer cells to promote pulmonary metastasis. J Biol Chem 283: 7616-7627, 2008.

17. Mitra AK, Sawada K, Tiwari P, Mui K, Gwin K and Lengyel E: Ligand-independent activation of c-Met by fibronectin and $\alpha(5)$ $\beta(1)$-integrin regulates ovarian cancer invasion and metastasis. Oncogene 30: 1566-1576, 2011.

18. Pontiggia O, Sampayo R, Raffo D, Motter A, Xu R, Bissell MJ, Joffé EB and Simian M: The tumor microenvironment modulates tamoxifen resistance in breast cancer: A role for soluble stromal factors and fibronectin through $\beta 1$ integrin. Breast Cancer Res Treat 133: 459-471, 2012.

19. Levine AJ: p53, the cellular gatekeeper for growth and division. Cell 88: 323-331, 1997.

20. Issaeva N, Bozko P, Enge M, Protopopova M, Verhoef LG, Masucci M, Pramanik A and Selivanova G: Small molecule RITA binds to p53, blocks p53-HDM-2 interaction and activates p53 function in tumors. Nat Med 10: 1321-1328, 2004.

21. Espinoza-Fonseca LM: Targeting MDM2 by the small molecule RITA: Towards the development of new multi-target drugs against cancer. Theor Biol Med Model 2: 38, 2005.

22. Enge M, Bao W, Hedström E, Jackson SP, Moumen A and Selivanova G: MDM2-dependent downregulation of p21 and hnRNP K provides a switch between apoptosis and growth arrest induced by pharmacologically activated p53. Cancer Cell 15: 171-183, 2009.

23. Grinkevich VV, Nikulenkov F, Shi Y, Enge M, Bao W, Maljukova A, Gluch A, Kel A, Sangfelt O and Selivanova G: Ablation of key oncogenic pathways by RITA-reactivated p53 is required for efficient apoptosis. Cancer Cell 15: 441-453, 2009.

24. Zhao CY, Grinkevich VV, Nikulenkov F, Bao W and Selivanova G: Rescue of the apoptotic-inducing function of mutant p53 by small molecule RITA. Cell Cycle 9: 1847-1855, 2010. 
25. Oh SJ, Jung SP, Han J, Kim S, Kim JS, Nam SJ, Lee JE and Kim JH: Silibinin inhibits TPA-induced cell migration and MMP-9 expression in thyroid and breast cancer cells. Oncol Rep 29: 1343-1348, 2013.

26. Yoon JH, Choi YJ and Lee SG: Ginsenoside Rh1 suppresses matrix metalloproteinase-1 expression through inhibition of activator protein-1 and mitogen-activated protein kinase signaling pathway in human hepatocellular carcinoma cells. Eur J Pharmacol 679: 24-33, 2012.
27. Kikkawa U, Takai Y, Tanaka Y, Miyake R and Nishizuka Y: Protein kinase $\mathrm{C}$ as a possible receptor protein of tumorpromoting phorbol esters. J Biol Chem 258: 11442-11445, 1983.

28. Robbins D, Ponville J, Morris K and Zhao Y: Involvement of PTEN in TPA-mediated p53-activation in mouse skin epidermal JB6 cells. FEBS Lett 586: 4108-4113, 2012.

29. Ashcroft M, Taya Y and Vousden KH: Stress signals utilize multiple pathways to stabilize p53. Mol Cell Biol 20: 3224-3233, 2000. 